\title{
Bosutinib in the management of chronic myelogenous leukemia
}

\author{
This article was published in the following Dove Press journal: \\ Biologics:Targets and Therapy \\ 3 May 2013 \\ Number of times this article has been viewed
}

\author{
Gunhild Keller-von Amsberg \\ Philippe Schafhausen \\ Department of Hematology \\ and Oncology and, Stem Cell \\ Transplantation and Pulmonology \\ Division, Oncological Center, \\ University Hospital Hamburg- \\ Eppendorf, Hamburg, Germany
}

\begin{abstract}
Bosutinib (SKI-606) is an orally available, once-daily dual Src and Abl kinase inhibitor, approved by the US Food and Drug Administration for the treatment of adults with chronic, accelerated, or blast-phase Philadelphia chromosome-positive chronic myelogenous leukemia who are intolerant of or resistant to first- or second-generation tyrosine kinase inhibitors. Bosutinib effectively overcomes the majority of imatinib-resistance-conferring BCR-ABL mutations except V299L and T315I. In the Bosutinib Efficacy and Safety in chronic myeloid LeukemiA (BELA) trial, bosutinib attained a faster and deeper molecular response than imatinib in newly diagnosed chronic-phase chronic myelogenous leukemia patients. Treatment-emergent adverse events are usually very manageable. Low grade, mostly self-limiting diarrhea represents the most frequently observed toxicity of bosutinib. Anti-diarrheal drugs, antiemetic agents, and/or fluid replacement should be used to treat these patients. The improved hematological toxicity of bosutinib compared with other tyrosine kinase inhibitors has been ascribed to its minimal activity against plateletderived growth factor receptor and KIT. In this review, we give an overview on the profile of bosutinib, the clinical potential and treatment-emergent adverse events.
\end{abstract}

Keywords: CML, BCR-ABL, SRC/ABL kinase inhibitor, resistance-conferring mutation

\section{Introduction}

The treatment landscape for chronic myelogenous leukemia (CML) was dramatically changed after the US Food and Drug Administration (FDA) approval of the tyrosine kinase inhibitor (TKI) imatinib (IM; formerly STI571 or CGP57148). ${ }^{1-4}$ IM effectively targets bcr-abl - a fusion protein resulting from the reciprocal translocation of the proto-oncogene $c$ - $A B L$ localized on chromosome 9 to the breakpoint cluster region $(B C R)$ on chromosome $22 .{ }^{5}$ In the International Randomized Interferon versus STI571 (IRIS) trial, the 8-year follow-up data revealed an estimated overall survival of $85 \%$ for IM, suggesting a high and persistent efficacy of this TKI in chronic-phase (CP) CML. ${ }^{6}$ However, due to intolerance and/or resistance, only $55 \%$ to $75 \%$ of the patients in the clinical trials are still on IM therapy after 5 to 8 years. The off-target effects of IM, with inhibition of platelet-derived growth factor receptor, kit, and abl kinases, mediate at least some of the toxicities ${ }^{7}$ and the BCR-ABL kinase-domain mutations that prevent IM binding are the main causes of IM resistance. ${ }^{8}$ Alternative treatment strategies are under investigation to address these important therapeutic issues.

The second-generation TKIs nilotinib (formerly AMN107) and dasatinib (formerly BMS354825) and third-generation TKI bosutinib (formerly SKI-606) have been found capable of overcoming the majority of IM-resistance-conferring BCR-ABL mutations. Convincing results of clinical trials led to the approval of nilotinib, dasatinib, and 
bosutinib for the treatment of CML patients, resistant or intolerant to IM. ${ }^{9-11}$

Due to the high efficacy of nilotinib, dasatinib, and bosutinib in preclinical and clinical studies, each compound was compared with IM in Phase III clinical trials for patients with newly diagnosed, untreated CP-CML. In all trials, nilotinib, dasatinib, and bosutinib were found to be superior to IM in terms of molecular response rate, time to the accomplishment of the remissions, and frequency of clonal evolution to accelerated phase (AP) and/or blast crisis (BC). ${ }^{12-14}$ However, direct comparisons between the trials are not admissible because of the distinct differences in trial design - in particular, regarding the primary study endpoints as well as different definitions for "freedom from progression," "event-free survival," and "progression-free survival."15

In this review, we give an overview of the clinical potential of bosutinib, with a special focus on treatmentemergent adverse events and the potential role of bosutinib in the future.

\section{Drug class}

Bosutinib is a third-generation TKI with the chemical name 3-quinolinecarbonitrile, 4-[(2,4-dichloro-5-methoxyphenyl) amino]-6-methoxy-7-[3-(4-methyl-1-piperazinyl)propoxy]-, hydrate (1:1). The chemical formula is $\mathrm{C}_{26} \mathrm{H}_{29} \mathrm{Cl}_{2} \mathrm{~N}_{5} \cdot \mathrm{H}_{2} \mathrm{O}$. Bosutinib can also be classified as a histone deacetylase inhibitor and induces differentiation and/or cell death of malignant cells. ${ }^{16}$

\section{Dosage, administration, and pharmacokinetics}

The recommended dose of bosutinib for CML patients is $500 \mathrm{mg}$ orally, taken once daily with food. Bosutinib is mainly metabolized by cytochrome P450 (CYP) $3 \mathrm{~A} 4{ }^{17}$ and is primarily excreted in the feces. Under feeding conditions, bosutinib exposure is linear and dose proportional over the entire evaluated dosage range, from 200 to $800 \mathrm{mg} .{ }^{18} \mathrm{In}$ healthy adult subjects, median time to peak plasma concentration is approximately 6 hours and mean terminal elimination half-life is $32-39$ hours. ${ }^{19}$

\section{Target profile of bosutinib}

Bosutinib shows potent inhibitory activity against src and bcr-abl, while activity against other kinases is low. ${ }^{20}$ Thus, Bosutinib virtually abrogates tyrosine phosphorylation of bcr-abl at concentrations of 25 to $50 \mathrm{nM}$ whereas $200 \mathrm{nM}$ is needed to decrease Abelson murine leukemia viral oncogene homolog 1 (v-abl1) phosphorylation to a similar extent. ${ }^{21}$
In addition, bosutinib effectively targets SRC at nanomolar concentrations with an half maximal inhibitory concentration (IC50) of $1.2 \mathrm{nM}$ in SRC enzymatic assay (bosutinib corresponds to compound 31a). ${ }^{22}$ This is of special interest because the SRC family kinases have been linked to disease progression and BCR-ABL-independent forms of IM resistance (reviewed in $\mathrm{Li}^{23}$ ). Additionally, in chemical proteomics and in vitro kinase assays, more than 45 potential target tyrosine and serine/threonine kinases of bosutinib were discovered. Apoptosis-linked STE20 kinases and CAMK2G - both associated with myeloid leukemia cell proliferation - were among these kinases. ${ }^{24}$ Further, bosutinib decreases activity of cyclin-dependent kinase 2, which leads to a prominent G1 arrest of clonal hematopoietic progenitors expressing BCR-ABL. ${ }^{25}$

\section{Clinical potential of bosutinib}

Bosutinib is effective in upfront treatment of CP-CML and after failure of first- and second-generation TKIs. It can overcome most IM-resistant $B C R-A B L$ mutations.

\section{First-line therapy for CP-CML}

In the open-label, randomized, multinational, Phase III Bosutinib Efficacy and Safety in chronic myeloid LeukemiA (BELA) trial, bosutinib (500 mg/d) was compared with IM (400 mg/d) for first-line treatment of CP-CML. A total of 502 patients were randomly assigned (1:1) to the two treatment groups after stratification for Sokal risk score and geographic region of the centers the patients were enrolled in for the study. ${ }^{12}$

Bosutinib did not meet the primary endpoint of the study because in the intention-to-treat population, complete cytogenetic response (CCyR) at 12 months was not significantly different for bosutinib (70\%; 95\% confidence interval [CI]: 64\%-76\%) when compared with IM (68\%; 95\% CI: $62 \%-74 \% ; P=0.601)$.

However, secondary endpoints as well as additional findings of the study suggest bosutinib is superior. Thus, the rate of major molecular remission (MMR) at 12 months was significantly higher in bosutinib-treated patients $(41 \% ; 95 \%$ CI: $35 \%-47 \%)$ than in IM-treated patients $(27 \% ; 95 \% \mathrm{CI}$ : $22 \%-33 \% ; P<0.001)$. Additionally, complete molecular remission was reported for $12 \%$ of bosutinib-treated patients but for only $3 \%$ of IM-treated individuals $(P<0.001)$ and the median time to CCyR and MMR was significantly shorter for bosutinib - 12.9 weeks (95\% CI: 12.6-13.4 weeks) and 37.1 weeks (95\% CI: 36.1-48.6 weeks), respectively - than with IM (24.6 weeks [95\% CI: 24.3-25.6 weeks, $P<0.001]$ and 72.3 weeks [95\% CI: 61.1 weeks-not reached, $P<0.001$ ], 
respectively). Most importantly, on-treatment transformation to AP or BC occurred in only four patients $(2 \%)$ treated with bosutinib, whereas this occurred in ten patients on IM (4\%). A total of three and eight CML-related deaths were reported for the bosutinib and the IM treatment arm, respectively. ${ }^{12}$

\section{Second-line therapy after failure of imatinib}

The efficacy of bosutinib secondary to treatment failure of IM in CP-CML was examined in a Phase I/II, nonrandomized clinical trial. The study population included imatinib-resistant $(\mathrm{n}=200)$ and imatinib-intolerant $(\mathrm{n}=88)$ patients. No other previous TKI exposure was allowed. "IM resistance" was defined as no hematological improvement within 4 weeks, no complete hematological response (CHR) by 3 months, no cytogenetic response by 6 months, or no major cytogenetic response (MCyR) by 12 months, with a daily IM dosage of at least $600 \mathrm{mg}$. "IM intolerance" was defined as grade IV hematological toxicity lasting for more than 7 days, appearance of grade 3 and 4 non-hematological toxicities, or grade 2 toxicities that did not improve despite adequate management or dose adjustments, or when a loss of previously attained response was observed after toxicity determined dose reduction of IM. ${ }^{11}$

Thirty-one percent of the patients achieved a MCyR at 24 weeks, thus met the primary endpoint of the study (33\% of IM-resistant patients; $27 \%$ of IM-intolerant patients). MCyR and CCyR were observed in $53 \%$ and $41 \%$ of patients, respectively, after a median follow-up time of 24.2 months. Dose intensities exceeding $350 \mathrm{mg}$ were associated with increased rates of MCyR. Within a median time of 2 weeks, $86 \%$ of the patients achieved a CHR, although $78 \%$ of study participants did not have a CHR at the time of study enrollment. Among patients with CCyR, MMR and complete molecular remission were reported for $64 \%$ and $49 \%$ of IM-resistant and $65 \%$ and $61 \%$ of IM-intolerant patients, respectively. ${ }^{11}$

\section{Third- and fourth-line therapy after failure of IM and nilotinib and/or dasatinib}

The clinical potential of bosutinib in third- or fourth-line therapy was examined in a subgroup of patients $(n=118)$ of the just-described multicenter clinical trial. ${ }^{26}$ All patients were previously treated with IM and had failed secondary therapy with nilotinib and/or dasatinib: 27 and 37 patients were resistant to nilotinib and dasatinib while intolerance was documented for one patient receiving nilotinib and 50 patients receiving dasatinib. Two patients were resistant to dasatinib and nilotinib, while one patient was intolerant to both second-generation TKIs.
The median duration of follow-up was 28.5 months (range: 0.3-56.2 months) at the data cutoff date of March 28, 2011. At this time, the median time of bosutinib treatment was 8.3 months (range: $0.2-51.8$ months). Of the patients, $32 \%$ attained a MCyR and 24\% attained CCyR, including one patient who achieved CCyR and had previously received all three TKIs.

A confirmed CHR was achieved or maintained by $73 \%$ of the patients including $65 \%$ of patients who did not have a $\mathrm{CHR}$ at baseline. Remarkably, two of three patients who were treated with all three TKIs had a confirmed CHR. Among 20 patients who showed inadequate treatment responses, dose escalation to $600 \mathrm{mg} / \mathrm{d}$ subsequently resulted in responses in $30 \%$ of the individuals, with three cases of CCyR. Ontreatment transformation to AP was observed in five patients after 16 to 428 days on study, while no patient transformed to $\mathrm{BC} .{ }^{26,27}$ To identify the group of patients that might respond best to bosutinib treatment, baseline characteristics of patients were analyzed. However, only minor cytogenetic response prior to dasatinib and/or nilotinib treatment and a lower percentage of Philadelphia chromosome-positive cells at baseline were found to be consistently predictive of better outcomes on bosutinib. ${ }^{28}$

\section{Efficacy of bosutinib against IM-resistance-conferring BCR-ABL mutations}

Structural and spectroscopic analyses have helped to understand the activity of bosutinib against IM-resistant $B C R-A B L$ mutants. ${ }^{29}$ Preclinical activity of bosutinib has been reported against most IM-resistant mutants of $B C R-A B L$ with the exception of T315I and V299L. ${ }^{20,30}$

In clinical studies, the impact of preexisting $B C R-A B L$ mutations on bosutinib efficacy was evaluated in patients pretreated with IM only $(n=115)$ or with IM followed by nilotinib and/or dasatinib $(n=39)$. Similar rates of CHR and MCyR were observed in patients with and without mutations and broadly across all $B C R-A B L$ mutations, including in individuals harboring the dasatinib-resistant F317L or the nilotinib-resistant $\mathrm{Y} 253 \mathrm{H}$ and $\mathrm{F} 359 \mathrm{C} / \mathrm{I} / \mathrm{V}$ mutations but not T315I (Table 1). When patients with T315I at baseline were excluded from the second-line setting, response rates for the remaining patients with $\geq 1$ mutation were $93 \%$ for CHR and $62 \%$ for MCyR. ${ }^{31}$ During second-line therapy, 18 patients who discontinued treatment had one or more new $B C R-A B L$ mutations (T315I, $\mathrm{n}=8$; V299L, $\mathrm{n}=3$; E255V, E450A, E450G, G250E, K378E, L273M, and $\mathrm{M} 244 \mathrm{~V}, \mathrm{n}=1 \mathrm{each}$ ) and, indeed, for 15 of these individuals, 
Table I Efficacy of bosutinib against BCR-ABL mutations in clinical studies

\begin{tabular}{|c|c|c|c|c|}
\hline \multirow{2}{*}{$\begin{array}{l}\text { Mutation } \\
\text { at baseline } \\
\text { Location } \\
\text { of mutation }\end{array}$} & \multicolumn{4}{|c|}{ Pretreatment } \\
\hline & \multicolumn{2}{|l|}{ Imatinib } & \multicolumn{2}{|c|}{$\begin{array}{l}\text { Imatinib + nilotinib } \\
\text { and/or dasatinib }\end{array}$} \\
\hline & \multicolumn{2}{|l|}{ Response } & \multicolumn{2}{|c|}{ Response } \\
\hline & $\begin{array}{l}\text { CHR } \\
\left(n / n_{\text {evaluable }}\right)\end{array}$ & $\begin{array}{l}\text { MCyR } \\
\left(n / n_{\text {evaluable }}\right)\end{array}$ & $\begin{array}{l}\text { CHR } \\
\left(n / n_{\text {evaluable }}\right)\end{array}$ & $\begin{array}{l}\text { MCyR } \\
\left(n / n_{\text {evaluable }}\right)\end{array}$ \\
\hline
\end{tabular}

\begin{tabular}{|c|c|c|c|c|}
\hline \multicolumn{5}{|l|}{ P-loop } \\
\hline L248V & $3 / 3$ & $2 / 3$ & & \\
\hline G250E & $1 / 1$ & $2 / 2$ & $3 / 6$ & $0 / 5$ \\
\hline Y253F & $\mathrm{I} / \mathrm{I}$ & $0 / 1$ & & \\
\hline $\mathrm{Y} 253 \mathrm{H}$ & $\mathrm{I} / \mathrm{I}$ & $1 / 1$ & $5 / 6$ & $4 / 6$ \\
\hline E255K & $2 / 2$ & $1 / 2$ & $0 / 1$ & $0 / 1$ \\
\hline E255V & $\mathrm{I} / \mathrm{I}$ & $\mathrm{I} / \mathrm{I}$ & $1 / 1$ & $0 / 1$ \\
\hline \multicolumn{5}{|c|}{ Non-p-loop } \\
\hline M35IT & $3 / 3$ & $6 / 6$ & $\mathrm{I} / \mathrm{I}$ & $0 / 1$ \\
\hline F359V & $6 / 6$ & $4 / 7$ & $0 / 2$ & $1 / 2$ \\
\hline F3I7L & $0 / 0$ & $3 / 3$ & $4 / 8$ & $\mathrm{I} / 7$ \\
\hline T3I5I & $1 / 2$ & $0 / 3$ & $2 / 7$ & $0 / 6$ \\
\hline M244V & $2 / 2$ & $2 / 3$ & $3 / 3$ & $2 / 3$ \\
\hline D276G & $\mathrm{I} / \mathrm{I}$ & $0 / 1$ & & \\
\hline L298V & $\mathrm{I} / \mathrm{I}$ & $0 / 1$ & & \\
\hline F3III & $\mathrm{I} / \mathrm{I}$ & $1 / 1$ & & \\
\hline F3IIL & $\mathrm{I} / \mathrm{I}$ & $\mathrm{I} / \mathrm{I}$ & & \\
\hline N33IS & $0 / 0$ & $1 / 1$ & & \\
\hline E355G & $2 / 2$ & $1 / 2$ & & \\
\hline F359l & $\mathrm{I} / \mathrm{I}$ & $\mathrm{I} / \mathrm{I}$ & & \\
\hline L364P & $0 / 0$ & $\mathrm{I} / \mathrm{I}$ & & \\
\hline L387F & $0 / 0$ & $0 / 1$ & & \\
\hline H396P & $\mathrm{I} / \mathrm{I}$ & $2 / 2$ & & \\
\hline H396R & $\mathrm{I} / \mathrm{I}$ & $0 / 0$ & $0 / 1$ & $0 / 1$ \\
\hline D42IG & $\mathrm{I} / \mathrm{I}$ & $0 / 1$ & & \\
\hline $1432 \mathrm{~T}$ & $0 / 0$ & $0 / 1$ & & \\
\hline $\mathrm{E} 450 \mathrm{~V}$ & $\mathrm{I} / \mathrm{I}$ & $\mathrm{I} / \mathrm{I}$ & & \\
\hline E453K & $0 / 0$ & $1 / 1$ & & \\
\hline E453Q & $\mathrm{I} / \mathrm{I}$ & $1 / 1$ & & \\
\hline E453A & & & $\mathrm{I} / \mathrm{I}$ & $0 / 1$ \\
\hline $\mathrm{E} 459 \mathrm{~K}$ & $0 / 0$ & $0 / 1$ & & \\
\hline P480A & $0 / 0$ & $1 / 1$ & & \\
\hline V299L & & & $\mathrm{I} / 2$ & $0 / 2$ \\
\hline Q300R & & & $\mathrm{I} / \mathrm{I}$ & $\mathrm{I} / \mathrm{I}$ \\
\hline N336S & & & $1 / 1$ & $0 / 1$ \\
\hline F359C & & & $2 / 2$ & $1 / 2$ \\
\hline F359I & & & $2 / 2$ & $2 / 2$ \\
\hline L387F & & & $\mathrm{I} / \mathrm{I}$ & $0 / 1$ \\
\hline C475V & & & $1 / 1$ & $1 / I$ \\
\hline F486S & & & $0 / 1$ & $0 / 1$ \\
\hline Unknown & $3 / 3$ & $9 / 11$ & & \\
\hline
\end{tabular}

Unknown $\quad 3 / 3$

Note: Copyright 2013. Reproduced with permission of American Society of Hematology (ASH). Cortes JE, Kantarjian HM, Brümmendorf TH, et al. Safety and efficacy of bosutinib (SKI-606) in chronic phase Philadelphia chromosome-positive chronic myeloid leukemia patients with resistance or intolerance to imatinib. Blood. 201 I; I 8(I7):4567-4576" and Copyright 2013. Reproduced with permission of American Society of Hematology (ASH). Khoury HJ, Cortes JE, Kantarjian HM, et al. Bosutinib is active in chronic phase chronic myeloid leukemia after imatinib and dasatinib and/or nilotinib therapy failure. Blood. 2012; I I (I5):3403-34I2. ${ }^{26}$ the reason for discontinuation was disease progression or lack of efficacy. ${ }^{31}$ In the course of third- and fourth-line treatment with bosutinib, nine individuals developed new mutations (V299L, $\mathrm{n}=4$; L248V, $\mathrm{n}=2$; T315I, $\mathrm{n}=2$; F359C, $\mathrm{n}=1 ; \mathrm{G} 250 \mathrm{E}, \mathrm{n}=1$ ) and eight of these patients discontinued bosutinib because of progressive disease or unsatisfactory response. ${ }^{26}$

\section{Efficacy of bosutinib in CML patients without resistance- conferring mutations at baseline}

In the second-line setting, $42.5 \%$ of the patients with IM resistance did not harbor a mutation at baseline. In addition, in $53 \%$ of the patients who were classified as resistant to IM and nilotinib and/or dasatinib, no mutation was detected at initiation of the study medication. Similar rates of CHR and MCyR were observed for both subgroups when compared with individuals of the respective trial who did have a resistance-conferring mutation at baseline. ${ }^{11,26}$

\section{Safety and tolerability of bosutinib}

Bosutinib shows an acceptable safety profile in the first-, second- and third-line therapy of CML patients. ${ }^{18}$ Following, a selection of the most frequent treatment-emergent adverse events is summarized.

\section{Hematological toxicity}

It should be noted that bosutinib has a favorable hematological toxicity profile compared with other TKIs. Thus, grade 3/4 neutropenia appeared significantly less frequently in patients treated with bosutinib when compared with IM in upfront therapy (11\% vs $24 \%)$. This has been ascribed to the minimal inhibitory activity of bosutinib against c-kit. Anemia and thrombocytopenia occurred to similar extents in bosutinib- and IM-treated patients. ${ }^{12}$ In clinical trials, grade $3 / 4$ hematological toxicities increased with the number of prior TKI therapies. Thus, grade $3 / 4$ thrombocytopenia was reported for $14 \%, 25 \%$, and $26 \%$ and neutropenia for $11 \%$, $17 \%$, and $20 \%$ of the patients receiving first-, second- or third-line bosutinib therapy, respectively. Worsening of grade $3 / 4$ anemias was less pronounced and ranged between $6 \%$ and $12 \%$ for bosutinib-treated patients. ${ }^{11,12,26}$ These findings are in line with observations of clinical studies performed with other TKIs in second- and third-line CML treatment. ${ }^{15}$

\section{Gastrointestinal toxicity}

Low grade, mostly self-limiting diarrhea has been reported in up to $84 \%$ of patients treated with bosutinib ${ }^{11,12,26}$ and thus represents the most frequently observed toxicity of the drug. 
The diarrhea usually occurs within the first 4 weeks after treatment initiation, is typically of low grade, and self-limits within the first 2 to 3 months of treatment. Anti-diarrheal drugs, antiemetic agents, and/or fluid replacement should be used to treat these patients. ${ }^{16}$ However, in the BELA trial, up to $11 \%$ of patients developed grade $3 / 4$ diarrhea under bosutinib therapy. In the bosutinib treatment arm, the diarrhea required dose interruptions and reductions in $21 \%$ and $8 \%$ of patients, respectively. In contrast, dose reduction was not necessary in any of the IM-treated patients and in only $6 \%$ of IM-treated patients was the TKI temporarily discontinued. ${ }^{12}$ Other gastrointestinal toxicities of bosutinib include nausea (31\%-46\%, all grades), vomiting (32\%-40\%, all grades), and abdominal pain (11\%-22\%, all grades). ${ }^{11,12,26}$

\section{Bleeding disorders}

Bosutinib has a minor influence on platelet function, resulting in a distinctly lower number of bleeding complications compared with other TKIs. ${ }^{15}$ Thus, hemorrhagic events appeared in only $5 \%$ of bosutinib-treated patients (all grades) with only one grade 3 bleeding event. ${ }^{11}$ In contrast, for dasatinib, bleeding disorders (all grades) were reported for $40 \%$ of the patients, with $10 \%$ classified as grade $3 / 4 .{ }^{15,32,33}$

\section{Non-hematological laboratory abnormalities}

The most frequent non-hematological laboratory abnormalities include hyperglycemia, hypermagnesemia; elevation of alanine aminotransferase and aspartate aminotransferase, uric acid, creatinine, lipase, and alkaline phosphatase; hypocalcaemia; and hypophosphatemia. ${ }^{11,12,26}$ An increase in liver enzymes was associated with a significantly increased rate of treatment discontinuations under bosutinib treatment compared with IM in first-line treatment. However, despite the occurrence of liver function abnormalities, no permanent liver injury was observed in patients treated with bosutinib. ${ }^{12}$

Other non-hematological treatment-emergent adverse events in first-, second- and third-line therapy include rashes (20\%-34\%, all grades), fatigue (11\%-22\%, all grades), headache (11\%-25\%, all grades), upper respiratory infections and cough (12\%-16\%, all grades). ${ }^{11,12,26}$

\section{Bosutinib application in patients with chronic hepatic impairment}

Bosutinib is mainly metabolized by CYP3A4 and, indeed, in a drug interaction study, ketoconazole, a potent CYP3A4 inhibitor, increased the maximal plasma concentration and concentration-time curve of $100 \mathrm{mg}$ oral bosutinib by 5.2and 8.6-fold, respectively. ${ }^{17}$
Therefore, the pharmacokinetics of bosutinib were evaluated in patients with impaired liver function (ChildPugh A n =6; Child-Pugh B n=6; Child-Pugh C n =6) and compared to healthy matched controls $(n=9)$. Elimination half-life was increased from 55 hours in healthy subjects to 86 hours in Child-Pugh A-, 113 hours in Child-Pugh B-, and 111 hours in Child-Pugh C-class patients. In addition, an increase of peak plasma concentration of 1.52- to 2.42-fold was observed in patients with impaired liver function when compared with healthy controls. ${ }^{19}$ Thus, dose adjustment needs to be taken into account when administering bosutinib to patients with impaired liver function or - if it cannot be avoided - to patients receiving CYP3A4 inhibitors at the same time.

\section{Expert commentary}

Bosutinib is approved by the FDA for the treatment of CML secondary to failure of IM, dasatinib, and/or nilotinib. Although bosutinib did not meet the primary endpoint of the BELA trial, it demonstrated high efficacy in the first-line treatment of CP-CML. Thus, deeper molecular response rates were reported for bosutinib than for IM. In addition, times to CCyR and MMR were significantly shorter for bosutinib than for IM, indicating a more rapid debulking of tumor burden with bosutinib. ${ }^{12}$ It has been speculated that one reason for the equal CCyR rates of both TKIs at 12 months was the unexpectedly high number of bosutinib discontinuations and interruptions in the intention-to-treat population due to adverse events. ${ }^{12}$ In fact, according to the study protocol, patients who discontinued the study counted as non-responders in the final analyses. However, remarkably, an exposure-response relationship for bosutinib has been observed for patients with newly diagnosed CP-CML with regard to CCyR, MMR, and cumulative CHR at 1 year. $^{34}$

Bosutinib effectively targets most IM-resistanceconferring BCR-ABL mutations, with the exception of the highly resistant T315I (resistant to IM, dasatinib, nilotinib, and bosutinib) and V299L mutations (resistant to dasatinib and bosutinib). For patients who do not respond to first- or second-generation TKIs, allogeneic stem cell transplantation and interferon therapy are currently the only approved treatment modalities with the potential for life prolongation. It has been speculated that a growing selection pressure in TKI treatment will promote the appearance of T315I mutations. ${ }^{35-37}$ Novel compounds such as the aurora kinase inhibitors - including danusertib (formerly PHA-739358), ${ }^{38}$ homoharringtonine, ${ }^{39}$ and ponatinib ${ }^{40}$ - successfully target the T315I mutation. In fact, clinical trials are currently underway and have attained promising results (see O'Hare et $\mathrm{al}^{41}$ for a 
review of these). Most convincingly, ponatinib demonstrated high efficacy independently of mutational status (including the highly resistant T315I mutation) in a Phase II clinical trial with heavily pretreated CP-, AP-, and BC-CML patients as well as patients with Philadelphia chromosome-positive acute lymphoblastic leukemia. ${ }^{42}$ Consequently, ponatinib was approved by the FDA in December 2012 for the treatment of adult patients with CP-, AP-, or BC-CML resistant or intolerant to prior TKI therapy.

In first-line therapy, a progression to AP or BC was observed less frequently in bosutinib-, dasatinib- and nilotinib-treated patients than in IM-treated individuals. An improved bcr-abl binding - resulting in early and deep molecular responses and therefore a more pronounced reduction of tumor cell burden - seems to be of high impact. In addition, src inhibition may further contribute to improved disease control in bosutinib- and dasatinib-treated patients. Indeed, src family tyrosine kinases have been associated with disease progression in CML, for example, increased expression and/or activation of Hck and Lyn were reported for patients progressing to AP or BC. ${ }^{42}$

The minimal activity of bosutinib against platelet-derived growth factor receptor and c-kit and a different inhibition profile against other target kinases are probably the cause of bosutinib's different toxicity profile compared with IM, nilotinib, and dasatinib. ${ }^{15,24,43,44}$ Thus, grade $3 / 4$ neutropenia was observed significantly less frequently with bosutinib than with IM in the BELA trial. ${ }^{12}$ One reason for this seems to be that bosutinib has lower toxicity toward normal progenitor cells than IM. ${ }^{45,46}$ Other advantages of bosutinib ${ }^{11,12}$ over other TKIs include a reduced number of bleeding disorders and fluid retention issues. ${ }^{10,14,47-49}$ As previously outlined, the most dominant adverse events resulting from bosutinib treatment are gastrointestinal toxicities (mainly grade 1/2 diarrhea and nausea), which are mostly easily managed and self-limiting.

\section{Future perspectives}

The choice of the best treatment strategy for CML has become increasingly difficult due to the growing armada of approved TKIs supplemented by several pipeline medications. In addition, in a growing number of countries, the standardization of molecular monitoring according to the international scale and the continuous improvement of techniques for molecular monitoring has resulted in increasingly precise BCR-ABL values. However, this raises the question of the optimal time for a therapeutic intervention - for example when suboptimal responses according to the definition of the European LeukemiaNET are observed..$^{50}$
Different clinical observations suggest that MMR is associated with increased individual life expectancy. Thus, Marin et al found a significantly higher 8-year probability of survival for patients with BCR-ABL transcript levels $<9.84 \%$ when compared with individuals with BCRABL transcript levels above this value at 3 months. ${ }^{51}$ In addition, Hanfstein et al reported lower 5-year survival rates for IM-treated patients with BCR-ABL levels $>10 \%$ according to the international scale at 3 months. ${ }^{52}$

In the BELA trial, BCR-ABL/ABL ratios $<10 \%$ at 3 , 6 , and 9 months were associated with higher rates of MMR and CCyR at later time points of the study in bosutinib- and IM-treated patients. Remarkably, more patients treated with bosutinib than with IM fell below the $10 \%$ BCR-ABL/ ABL ratio. ${ }^{53}$ Similarly, in first-line trials with dasatinib (Dasatinib versus Imatinib Study in Treatment-Naive CML Patients [DASISION]) and nilotinib (Evaluating Nilotinib Efficacy and Safety in Clinical Trials Newly Diagnosed Patients [ENESTnd]), more patients treated with the secondgeneration TKIs achieved BCR-ABL/ABL ratios $<10 \%$ at 3 months than with IM..$^{54,55}$ Independent of the TKI treatment, better molecular responses were associated with improved long-term outcome and a lower risk of transformation to $\mathrm{AP} / \mathrm{BC}$.

\section{Conclusion}

Highly potent second- and third-generation TKIs may be the better choice for first-line therapy of patients with long life expectancy. In addition, in our opinion, a TKI switch should be considered if the BCR-ABL/ABL ratio exceeds $10 \% 3$ months after treatment initiation to avoid an unfavorable course of disease with an increased risk of disease progression. For the choice of the second- or third-line TKI, mutational analyses, the patient's coexisting medical conditions, and possible treatment-emergent adverse events are of high importance. Because of its distinctly different toxicity profile, bosutinib is a genuine alternative to IM, nilotinib, and dasatinib.

\section{Disclosure}

Philippe Schafhausen is a consultant/advisor for Novartis and Pfizer and both authors have received travel support and lecture honoraria from Bristol-Myers Squibb and Novartis. The authors declare no other conflicts of interest in this work.

\section{References}

1. Cortes J, Goldman JM, Hughes T. Current issues in chronic myeloid leukemia: monitoring, resistance, and functional cure. J Natl Compr Canc Netw. 2012;10 Supp1 3:S1-S13. 
2. Deininger MW, O`Brien SG, Ford JM, Druker BJ. Practical management of patients with chronic myeloid leukemia receiving imatinib. $J$ Clin Oncol. 2003;21(8):1637-1647.

3. O`Brien SG, Guilhot F, Goldman JM, et al. International Randomized Study of Interferon Versus STI571 (IRIS) 7-year follow-up: sustained survival, low rate of transformation and increased rate of major molecular response (MMR) in patients (pts) with newly diagnosed chronic myeloid leukemia in chronic phase (CMLCP) treated with imatinib (IM). ASH Annual Meeting Abstracts. 2008;Abstract 186.

4. Sawyers CL, Hochhaus A, Feldman E, et al. Imatinib induces hematologic and cytogenetic responses in patients with chronic myelogenous leukemia in myeloid blast crisis: results of a phase II study. Blood. 2002;99(10):3530-3539.

5. Rowley JD. Letter: a new consistent chromosomal abnormality in chronic myelogenous leukaemia identified by quinacrine fluorescence and Giemsa staining. Nature. 1973;243(5405):290-293.

6. Deininger M, Guilhot F, Goldman JM, et al. International Randomized Study of Interferon Vs STI571 (IRIS) 8-year follow up: sustained survival and low risk for progression or events in patients with newly diagnosed chronic myeloid leukemia in chronic phase (CMLCP) treated with imatinib. ASH Annual Meeting Abstracts. 2009; Abstract 1126.

7. Buchdunger E, Zimmermann J, Mett $\mathrm{H}$, et al. Inhibition of the Abl protein-tyrosine kinase in vitro and in vivo by a 2 -phenylaminopyrimidine derivative. Cancer Res. 1996;56(1):100-104.

8. Ernst T, Hochhaus A. Chronic myeloid leukemia: clinical impact of BCR-ABL1 mutations and other lesions associated with disease progression. Semin Oncol. 2012;39(1):58-66.

9. Kantarjian H, Giles F, Wunderle L, et al. Nilotinib in imatinib-resistant CML and Philadelphia chromosome-positive ALL. N Engl J Med. 2006; 354(24):2542-2551.

10. Hochhaus A, Kantarjian HM, Baccarani M, et al. Dasatinib induces notable hematologic and cytogenetic responses in chronic-phase chronic myeloid leukemia after failure of imatinib therapy. Blood. 2007;109(6): 2303-2309.

11. Cortes JE, Kantarjian HM, Brümmendorf TH, et al. Safety and efficacy of bosutinib (SKI-606) in chronic phase Philadelphia chromosome-positive chronic myeloid leukemia patients with resistance or intolerance to imatinib. Blood. 2011;118(17):4567-4576.

12. Cortes JE, Kim DW, Kantarjian HM, et al. Bosutinib versus imatinib in newly diagnosed chronic-phase chronic myeloid leukemia: results from the BELA trial. $J$ Clin Oncol. 2012;30(28):3486-3492.

13. Saglio G, Kim DW, Issaragrisil $\mathrm{S}$, et al; ENESTnd Investigators. Nilotinib versus imatinib for newly diagnosed chronic myeloid leukemia. $N$ Engl J Med. 2010;362(24):2251-2259.

14. Kantarjian H, Shah NP, Hochhaus A, et al. Dasatinib versus imatinib in newly diagnosed chronic-phase chronic myeloid leukemia. $N$ Engl J Med. 2010;362(24):2260-2270.

15. Keller-von Amsberg G, Koschmieder S. Profile of bosutinib and its clinical potential in the treatment of chronic myeloid leukemia. OncoTargets and Therapy. 2013 March Volume 2013;6:99-106.

16. Goldenberg MM. Pharmaceutical approval update. $P$ T. 2012;37(11): 620-649.

17. Abbas R, Hug BA, Leister C, Burns J, Sonnichsen D. Effect of ketoconazole on the pharmacokinetics of oral bosutinib in healthy subjects. J Clin Pharmacol. 2011;51(12):1721-1727.

18. Abbas R, Hug BA, Leister C, Gaaloul ME, Chalon S, Sonnichsen D. A phase I ascending single-dose study of the safety, tolerability, and pharmacokinetics of bosutinib (SKI-606) in healthy adult subjects. Cancer Chemother Pharmacol. 2012;69(1):221-227.

19. Abbas R, Chalon S, Leister C, Gaaloul ME, Sonnichsen D. Evaluation of the pharmacokinetics and safety of bosutinib in patients with chronic hepatic impairment and matched healthy subjects. Cancer Chemother Pharmacol. 2013;71(1):123-132.

20. Puttini M, Coluccia AM, Boschelli F, et al. In vitro and in vivo activity of SKI-606, a novel Src-Abl inhibitor, against imatinib-resistant BcrAbl+ neoplastic cells. Cancer Res. 2006;66(23):11314-11322.
21. Golas JM, Arndt K, Etienne C, et al. SKI-606, a 4-anilino-3quinolinecarbonitrile dual inhibitor of Src and Abl kinases, is a potent antiproliferative agent against chronic myelogenous leukemia cells in culture and causes regression of K562 xenografts in nude mice. Cancer Res. 2003;63(2):375-381.

22. Boschelli DH, Ye F, Wang YD, et al. Optimization of 4-phenylamino-3quinolinecarbonitriles as potent inhibitors of Src kinase activity. J Med Chem. 2001;44(23):3965-3977.

23. Li S. Src-family kinases in the development and therapy of Philadelphia chromosome-positive chronic myeloid leukemia and acute lymphoblastic leukemia. Leuk Lymphoma. 2008;49(1):19-26.

24. Remsing Rix LL, Rix U, Colinge J, et al. Global target profile of the kinase inhibitor bosutinib in primary chronic myeloid leukemia cells. Leukemia. 2009;23(3):477-485.

25. Mancini M, Brusa G, Zuffa E, et al. Persistent Cdk2 inactivation drives growth arrest of BCR-ABL-expressing cells in response to dual inhibitor of SRC and ABL kinases SKI606. Leuk Res. 2007;31(7): 979-987.

26. Khoury HJ, Cortes JE, Kantarjian HM, et al. Bosutinib is active in chronic phase chronic myeloid leukemia after imatinib and dasatinib and/or nilotinib therapy failure. Blood. 2012;119(15):3403-3412.

27. Khoury HJ, Gambacorti-Passerini C, Kantarjian HM, et al. Bosutinib as therapy for chronic phase chronic myeloid leukemia following failure with imatinib plus dasatinib and/or nilotinib: 24-month minimum follow-up update. ASH Annual Meeting Abstracts. 2012;Abstract:3785.

28. Cortes JE, Khoury HJ, Lipton JH, et al. Baseline predictors of response to bosutinib in patients with chronic phase chronic myeloid leukemia following resistance or intolerance to imatinib plus dasatinib and/or nilotinib. ASH Annual Meeting Abstracts. 2012;Abstract:2793.

29. Levinson NM, Boxer SG. Structural and spectroscopic analysis of the kinase inhibitor bosutinib and an isomer of bosutinib binding to the Abl tyrosine kinase domain. PLoS One. 2012;7(4):e29828.

30. Redaelli S, Piazza R, Rostagno R, et al. Activity of bosutinib, dasatinib, and nilotinib against 18 imatinib-resistant BCR/ABL mutants. J Clin Oncol. 2009;27(3):469-471.

31. Cortes JE, Kantarjian HM, Kim DW, et al. Bosutinib as therapy for chronic phase chronic myeloid leukemia following resistance or intolerance to imatinib: 36-month minimum follow-up update. ASH Annual Meeting Abstracts. 2012;Abstract:3779.

32. Brave M, Goodman V, Kaminskas E, et al. Sprycel for chronic myeloid leukemia and Philadelphia chromosome-positive acute lymphoblastic leukemia resistant to or intolerant of imatinib mesylate. Clin Cancer Res. 2008;14(2):352-359.

33. Quintás-Cardama A, Han X, Kantarjian H, Cortes J. Tyrosine kinase inhibitor-induced platelet dysfunction in patients with chronic myeloid leukemia. Blood. 2009;114(2):261-263.

34. Hsyu PH, Mould DR, Upton RN, Amantea M. Pharmacokineticpharmacodynamic relationship of bosutinib in patients with chronic phase chronic myeloid leukemia. Cancer Chemother Pharmacol. 2013;71(1):209-218.

35. Cortes J, Lipton JH, Rea D, et al; Omacetaxine 202 Study Group. Phase 2 study of subcutaneous omacetaxine mepesuccinate after TKI failure in patients with chronic-phase CML with T315I mutation. Blood. 2012;120(13):2573-2580.

36. Hughes T, Saglio G, Branford S, et al. Impact of baseline BCR-ABL mutations on response to nilotinib in patients with chronic myeloid leukemia in chronic phase. J Clin Oncol. 2009;27(25):4204-4210.

37. Soverini S, Colarossi S, Gnani A, et al. Resistance to dasatinib in Philadelphia-positive leukemia patients and the presence or the selection of mutations at residues 315 and 317 in the BCR-ABL kinase domain. Haematologica. 2007;92(3):401-404.

38. Gontarewicz A, Balabanov S, Keller G, et al. Simultaneous targeting of Aurora kinases and Bcr-Abl kinase by the small molecule inhibitor PHA-739358 is effective against imatinib-resistant BCR-ABL mutations including T315I. Blood. 2008;111(8):4355-4364.

39. Quintás-Cardama A, Cortes J. Homoharringtonine for the treatment of chronic myelogenous leukemia. Expert Opin Pharmacother. 2008;9(6): 1029-1037. 
40. Cortes JE, Kim DW, Pinilla-Ibarz J, et al; The PACE Study Group. Initial findings from the PACE trial: a pivotal phase 2 study of ponatinib in patients with CML and Ph+ALL resistant or intolerant to dasatinib or nilotinib, or with the t315i mutation. ASH Annual Meeting Abstracts. 2011;Abstract:109.

41. O`Hare T, Eide CA, Deininger MW. New Bcr-Abl inhibitors in chronic myeloid leukemia: keeping resistance in check. Expert Opin Investig Drugs. 2008;17(6):865-878.

42. Hu Y, Liu Y, Pelletier S, et al. Requirement of Src kinases Lyn, Hck and Fgr for BCR-ABL1-induced B-lymphoblastic leukemia but not chronic myeloid leukemia. Nat Genet. 2004;36(5):453-461.

43. Bantscheff M, Eberhard D, Abraham Y, et al. Quantitative chemical proteomics reveals mechanisms of action of clinical ABL kinase inhibitors. Nat Biotechnol. 2007;25(9):1035-1044.

44. Keller-V Amsberg G, Brümmendorf TH. Novel aspects of therapy with the dual Src and Abl kinase inhibitor bosutinib in chronic myeloid leukemia. Expert Rev Anticancer Ther. 2012;12(9):1121-1127.

45. Konig H, Holyoake TL, Bhatia R. Effective and selective inhibition of chronic myeloid leukemia primitive hematopoietic progenitors by the dual Src/Abl kinase inhibitor SK-606. Blood. 2008;111(4): 2329-2338.

46. Bartolovic K, Balabanov S, Hartmann U, et al. Inhibitory effect of imatinib on normal progenitor cells in vitro. Blood. 2004;103(2): $523-529$.

47. Gora-Tybor J, Robak T. Targeted drugs in chronic myeloid leukemia. Curr Med Chem. 2008;15(29):3036-3051.

48. Talpaz M, Shah NP, Kantarjian H, et al. Dasatinib in imatinibresistant Philadelphia chromosome-positive leukemias. $N$ Engl J Med. 2006;354(24):2531-2541
49. Quintás-Cardama A, Kantarjian H, O`Brien S, et al. Pleural effusion in patients with chronic myelogenous leukemia treated with dasatinib after imatinib failure. J Clin Oncol. 2007;25(25):3908-3914.

50. Lima L, Bernal-Mizrachi L, Saxe D, et al. Peripheral blood monitoring of chronic myeloid leukemia during treatment with imatinib, second-line agents, and beyond. Cancer. 2011;117(6):1245-1252.

51. Marin D, Ibrahim AR, Lucas C, et al. Assessment of BCR-ABL1 transcript levels at 3 months is the only requirement for predicting outcome for patients with chronic myeloid leukemia treated with tyrosine kinase inhibitors. J Clin Oncol. 2012;30(3):232-238.

52. Hanfstein B, Müller MC, Hehlmann R, et al; SAKK; German CML Study Group. Early molecular and cytogenetic response is predictive for long-term progression-free and overall survival in chronic myeloid leukemia (CML). Leukemia. 2012;26(9):2096-2102.

53. Brümmendorf TH, Kantarjian HM, Gambacorti-Passerini C, et al. Assessment of early molecular response as a predictor of long-term clinical outcomes in the phase 3 BELA study. ASH Annual Meeting Abstracts. 2012;Abstract:69.

54. Hochhaus A, Hughes TP, Saglio G, et al. Outcome of patients with chronic myeloid leukemia in chronic phase (CML-CP) based on early molecular response and factors associated with early response: 4-year follow-up data from ENESTnd (Evaluating Nilotinib Efficacy and Safety in Clinical Trials Newly Diagnosed Patients). ASH Annual Meeting Abstracts. 2012;Abstract:167.

55. Saglio G, Kantarjian HM, Shah N, et al. Early response (molecular and cytogenetic) and long-term outcomes in newly diagnosed chronic myeloid leukemia in chronic phase (CML-CP): exploratory analysis of DASISION 3-year data. ASH Annual Meeting Abstracts. 2012; Abstract:1675.

\section{Publish your work in this journal}

Biologics: Targets \& Therapy is an international, peer-reviewed journal focusing on the patho-physiological rationale for and clinical application of Biologic agents in the management of autoimmune diseases, cancers or other pathologies where a molecular target can be identified. This journal is indexed on PubMed Central, CAS, EMBase, Scopus

\section{Dovepress}

and the Elsevier Bibliographic databases. The manuscript management system is completely online and includes a very quick and fair peerreview system, which is all easy to use. Visit http://www.dovepress com/testimonials.php to read real quotes from published authors. 